\section{Perfiles de transición epidemiológica- -nutricional y carga de morbi-mortalidad por COVID-19 en Argentina: un estudio ecológico}

\author{
Profiles of epidemiological-nutritional transition \\ and burden of morbimortality from COVID-19 \\ in Argentina: an ecological study
}

\section{Perfis de transição epidemiológica-nutricional e carga de morbimortalidade por COVID-19 \\ na Argentina: um estudo ecológico}

\section{Resumen}

El objetivo de este trabajo fue identificar perfiles epidemiológico-nutricionales en Argentina, y comparar su carga de morbi-mortalidad por COVID-19. Se condujo un estudio ecológico multigrupal (24 unidades geográficas de Argentina). Se estimó el porcentaje de cambio 2005-2018 de indicadores de transición epidemiológica-nutricional, y sociodemográficos por unidad geográfica. Se condujo un análisis de componentes principales y análisis de clúster jerárquico para identificar agrupamientos geográficos que definan perfiles de transición epidemiológica-nutricional. Por grupo, se calcularon medidas resumen de incidencia acumulada (IA), mortalidad y tasa de letalidad por COVID-19 (semana epidemiológica 50), estableciendo comparaciones mediante prueba $t$ de Student. Se identificaron tres perfiles denominados: (1) reemergencia de enfermedades infecciosas, (2) persistencia de enfermedades cardiovasculares con mejoras sociosanitarias y en estilos de vida, y (3) consolidación de la tríada obesidad-sedentarismo-enfermedades cardiometabólicas. La IA y mortalidad por COVID-19 promedio fue mayor en provincias con perfil 1, en comparación con el perfil 2 (IA: $p=0,0159$; mortalidad: $p=0,0187$ ) y el perfil 3 (IA: $p=0,0205)$. La letalidad resultó mayor en el perfil 3, que aglomera provincias con situación socioeconómica más desfavorable, estimándose diferencias significativas respecto al perfil $2(p=0,0307)$. En conclusión, existen distintos perfiles epidemiológico-nutricionales en Argentina, que tienden a diferenciarse en su situación epidemiológica de COVID-19. Las estrategias contra COVID-19 deberían considerar las características epidemiológico-nutricionales y sociodemográficas de base.

Infecciones por Coronavirus; Transición Nutricional; Dinámica Poblacional; Epidemiología Social
Graciela Fabiana Scruzzi 1,2

Natalia Tumas 2,3

Sonia Alejandra Pou 1,4

\author{
Correspondencia \\ S. A. Pou \\ Instituto de Investigaciones en Ciencias de la Salud, Consejo \\ Nacional de Investigaciones Científicas y Técnicas/Universidad \\ Nacional de Córdoba, Córdoba, Argentina. \\ Bv. de la Reforma y Enfermera Gordillo, Ciudad Universitaria, \\ Córdoba 5016, Argentina. \\ pousonia@conicet.gov.ar
1 Facultad de Ciencias Médicas, Universidad Nacional de Córdoba, Córdoba, Argentina.
2 Facultad de Ciencias de la Salud, Universidad Católica de Córdoba, Córdoba, Argentina.
3 Centro de Investigaciones y Estudios sobre Cultura y Sociedad, Consejo Nacional de Investigaciones Científicas y Técnicas/ Universidad Nacional de Córdoba, Córdoba, Argentina. Nacional de Investigaciones Científicas y Técnicas/Universidad Nacional de Córdoba, Córdoba, Argentina. \\ 4 Instituto de Investigaciones en Ciencias de la Salud, Consejo
}




\section{Introducción}

La teoría de la transición demográfica, postulada a inicios del siglo XX para explicar la dinámica demográfica de los países desarrollados, describe cambios en los patrones de mortalidad y natalidad ligados al proceso de industrialización y modernización que atraviesan las sociedades 1. Esta teoría sentó las bases para el desarrollo de otras con mirada "transicional", como la transición epidemiológi$\mathrm{ca}^{2} \mathrm{y}$ nutricional 3 , que han servido de marco para analizar los cambios en los perfiles epidemiológico-nutricionales de las poblaciones, aunque no siempre de manera integrada.

En la teoría de transición epidemiológica, Omran 2 describe estadios que dan cuenta de cómo las enfermedades no transmisibles fueron superando a las enfermedades transmisibles (infecciosas) como principal carga relativa de morbi-mortalidad. Más tarde, atendiendo a otras experiencias regionales, fueron descritas nuevas características del proceso $4,5,6,7,8$, incluido un estadio avanzado de coexistencia de enfermedades emergentes (VIH, hepatitis B y C), con otras resurgentes (tuberculosis, cólera), junto a cambios saludables en los estilos de vida y decrecimiento de la mortalidad por enfermedades cardiovasculares. Para países en desarrollo, ya en 1998 , $\operatorname{Omran}^{9}$ (p. 105) reconoce una fase denominada "la edad de la triple carga de salud", con persistencia de antiguos problemas de salud (enfermedades transmisibles, morbi-mortalidad materna, deficiencias nutricionales), incremento de otros nuevos (enfermedades crónicas, estrés y enfermedades por causas externas), y existencia de sistemas de salud inadecuadamente preparados para ello.

La transición nutricional, estrechamente ligada a la epidemiológica, constituye un proceso de cambios en los patrones de alimentación y actividad física, determinados por fenómenos socioeconómicos, demográficos y socioculturales interrelacionados 10,11. Popkin 12 describió sus fases, desde una etapa temprana caracterizada por una dieta basada en la recolección de alimentos, estilo de vida activo y predominio de infecciones, a una etapa avanzada con una dieta "occidental" (caracterizada por un alto contenido de grasas saturadas, azúcares, y alimentos refinados), mayor sedentarismo y predominio de enfermedades crónicas-degenerativas y obesidad. En Argentina, se ha indicado la coexistencia de escenarios socio-nutricionales diversos, que evidencian gran heterogeneidad interna en este proceso 13 . El presente trabajo parte de la idea de "transiciones poblacionales" múltiples (epidemiológicas, nutricionales, demográficas) e interrelacionadas, espacial y temporalmente determinadas. Se adhiere a la idea de que la "transición epidemiológica nutricional" constituye un componente a valorar conjuntamente en el marco transicional 14.

En el escenario actual, un aspecto adicional a considerar es que la irrupción de la pandemia de COVID-19 pone en tensión el clásico enfoque de transición epidemiológica, que promulga la confluencia generalizada de los países a perfiles epidemiológicos con altas cargas de enfermedades degenerativas, en detrimento de las infecciosas. La pandemia de COVID-19 emerge además con persistencia de la obesidad como problemática creciente, a su vez asociada a la severidad del COVID-19 15,16. Esta situación epidemiológica sin precedentes abre nuevos interrogantes que obligan a considerar marcos transicionales amplios.

La situación conocida del COVID-19 en Argentina ya evidencia desigualdades geográficas importantes. Como hipótesis, suponemos que estas desigualdades en la carga de morbi-mortalidad por COVID-19 podrían relacionarse con la existencia de distintos perfiles transicionales epidemiológico-nutricionales de las poblaciones, previos a la pandemia. Esto se basa, por un lado, en evidencia que indica que en este país coexisten diferentes estadios de transición nutricional, estrechamente relacionados con sus características sociodemográficas de base 13. Por otra parte, toma en consideración el reciente consenso de la comunidad científica sobre que comorbilidades, como obesidad y diabetes, conllevan un peor pronóstico de COVID-19 15,16. Siendo que, a nivel epidemiológico, una carga relativa creciente de estas patologías crónicas no transmisibles supone estadios más avanzados en los procesos transicionales epidemiológico y nutricional, podemos suponer que el impacto de la pandemia puede ser distinto según el estadio o fase transicional que identifique a cada población. Así, el objetivo de este trabajo fue identificar los perfiles epidemiológico-nutricionales, de manera integrada, que caracterizan a la población argentina en el periodo 2005-2018, y analizar si existen diferencias preliminares entre estos en su carga de morbi-mortalidad por COVID-19, considerando un enfoque transicional. 


\section{Materiales y métodos}

\section{Tipo de estudio y fuentes de datos}

Se realizó un estudio ecológico multigrupal (24 unidades geográficas, correspondientes a 23 provincias más Ciudad Autónoma de Buenos Aires) en Argentina. Se utilizaron los reportes oficiales de la Encuesta Nacional de Factores de Riesgo (ENFR) 2005 y 2018, y anuarios de la Dirección de Estadísticas e Información en Salud (DEIS) 2007 y 2019. Los datos referidos a COVID-19, por cada unidad geográfica, fueron obtenidos del Sistema de Información sobre COVID-19 para la Región de las Américas de Organización Panamericana de la Salud (Geo-Hub COVID-19 - Information System for the Region of the Americas. https://paho-covid19-response-who.hub.arcgis.com/pages/paho-argentina-covid19-response, accedido el 08/Dic/2020).

\section{Variables}

Para la caracterización de los perfiles epidemiológico-nutricionales, las variables de interés se organizaron según los ejes: transición epidemiológica, transición nutricional, y otros indicadores sociodemográficos, como muestra el Cuadro 1. Como la transición per se supone una dinámica temporal, se construyó, para cada variable, dos nuevas correspondientes a la diferencia absoluta y relativa (\% de cambio) de los valores registrados en los años extremos disponibles, dentro del periodo 2005-2018. Como indicadores de carga de morbi-mortalidad por COVID-19, se seleccionaron la tasa de incidencia acumulada (casos confirmados por 100.000 personas), mortalidad acumulada (defunciones totales por 100.000 personas) y tasa de letalidad (\% de defunciones sobre el total de casos confirmados), correspondientes a la semana epidemiológica 50 (al 8 de diciembre de 2020).

\section{Análisis de datos}

Preliminarmente, se estimaron medidas resumen a nivel nacional por cada variable en 2005 y 2018, analizando diferencias entre años mediante pruebas de hipótesis para datos apareados (prueba $t$ de Student de diferencias de medias, o prueba de los rangos con signo de Wilcoxon, según se cumpla o no el supuesto de normalidad, respectivamente). Se calcularon también las diferencias promedio 2005-2018 (absolutas y relativas).

La identificación de perfiles epidemiológico-nutricionales se condujo en dos etapas: primero, se empleó un análisis de componentes principales (ACP) para obtener componentes (dimensiones) que sinteticen las principales relaciones entre variables; luego, tomando como base los componentes del ACP, se aplicó un análisis de clúster jerárquico (aglomerativo, por método de Ward) para identificar agrupamientos geográficos (conglomerados) que definan distintos perfiles de transición epidemiológica-nutricional en Argentina.

En el ACP se incluyeron como variables activas (que participan en la construcción de los componentes principales), los porcentajes de cambio 2005-2018 de los indicadores de transición epidemiológica y nutricional, y como inactivas (variables que sólo son proyectadas para facilitar su interpretación) los correspondientes al eje sociodemográfico (Cuadro 1). Para definir el número de componentes principales a interpretar se consideró la variabilidad explicada $(>60 \%)$ y el criterio de autovalores $(>1)$.

El análisis de clúster permitió agrupar a las unidades de análisis (provincias y Ciudad Autónoma de Buenos Aires), en base a la medición de las distancias en el espacio construido por el ACP. Mediante dendograma se construyó una partición óptima de la base de datos en tres agrupamientos que conformaron los diferentes perfiles identificados.

La información sobre COVID-19 fue descrita mediante cálculo de medidas resumen en cada agrupamiento (perfil). Las distribuciones de los indicadores de morbi-mortalidad por COVID-19 en cada grupo fueron analizadas mediante teste de Shapiro-Wilk para contrastar la normalidad de los datos. Siendo que en todos los casos se aceptó la hipótesis nula de normalidad (con un nivel de significación igual a 0,05), se empleó como método de comparación de medias la prueba de hipótesis t de Student a 


\section{Cuadro 1}

Variables, indicadores y fuentes de datos seleccionados según ejes de estudio. Argentina, 2005-2018.

\begin{tabular}{|c|c|c|}
\hline VARIABLE & INDICADOR & FUENTE DE DATOS \\
\hline \multicolumn{3}{|c|}{ Transición epidemiológica } \\
\hline Mortalidad infantil & $\begin{array}{c}\text { Tasa de mortalidad infantil por } 1.000 \text { nacidos } \\
\text { vivos. }\end{array}$ & Anuario DEIS 2007 y 2019 * \\
\hline APVP por causas cardiovasculares & $\begin{array}{l}\text { Años potenciales de vida perdidos por causas } \\
\text { cardiovasculares (0-70 años) cada } 10.000 \\
\text { habitantes. CIE-10 I00 al 199, excepto } 146 .\end{array}$ & Anuario DEIS 2007 y 2019 * \\
\hline APVP por tumores & $\begin{array}{c}\text { Años potenciales de vida perdidos por } \\
\text { tumores (0-70 años) cada } 10.000 \text { habitantes. } \\
\text { CIE-10 C00 a D48. }\end{array}$ & Anuario DEIS 2007 y 2019 * \\
\hline APVP por causas externas & $\begin{array}{l}\text { Años potenciales de vida perdidos por causas } \\
\text { externas (0-70 años) cada } 10.000 \text { habitantes. } \\
\text { CIE-10 V01 a V99; W00 a Y98 (incluye } \\
\text { accidentes, suicidios y homicidios). }\end{array}$ & Anuario DEIS 2007 y 2019 * \\
\hline APVP por causas infecciosas & $\begin{array}{c}\text { Años potenciales de vida perdidos por } \\
\text { enfermedad infecciosa (0-70 años) cada } 10.000 \\
\text { habitantes. CIE-10 A00 a B99; J00 a J22; } \\
\text { G00 a G03. }\end{array}$ & Anuario DEIS 2007 y 2019 * \\
\hline Tasa de sífilis congénita & $\begin{array}{l}\text { Tasa de incidencia acumulada de sífilis } \\
\text { congénita por } 100.000 \text { nacidos vivos. }\end{array}$ & Anuario DEIS 2007 y 2019 * \\
\hline Prevalencia de hipertensión arterial & $\begin{array}{c}\text { Porcentaje de población adulta que refiere } \\
\text { estar en tratamiento por hipertensión arterial. }\end{array}$ & ENFR 200534 y 201835 \\
\hline Prevalencia de diabetes tipo 2 & $\begin{array}{l}\text { Porcentaje de población adulta que refiere } \\
\text { estar en tratamiento por diabetes tipo } 2 \text {. }\end{array}$ & ENFR 200534 y 201835 \\
\hline \multicolumn{3}{|c|}{ Transición nutricional } \\
\hline Bajo nivel de actividad física & $\begin{array}{l}\text { Porcentaje de población adulta con bajo nivel } \\
\text { de actividad física. }\end{array}$ & ENFR 200534 y 201835 \\
\hline Obesidad en adultos/as & $\begin{array}{l}\text { Porcentaje de personas de } 18 \text { años o más con } \\
\text { IMC autorreportado igual o superior a } 30 .\end{array}$ & ENFR 200534 y 201835 \\
\hline Bajo peso al nacer & $\begin{array}{l}\text { Porcentaje de nacidos/as vivos/as con peso } \\
\text { inferior a } 2.500 \text { gramos. }\end{array}$ & Anuario DEIS 2007 y 2019 * \\
\hline \multicolumn{3}{|c|}{ Indicadores sociodemográficos } \\
\hline Población sin cobertura de salud & $\begin{array}{l}\text { Porcentaje de población sin cobertura de obra } \\
\text { social o plan médico. }\end{array}$ & Anuario DEIS 2007, 2019 en base a datos censales * \\
\hline Esperanza de vida al nacer & Esperanza de vida al nacer. & Anuario DEIS 2007, 2019 \\
\hline $\begin{array}{l}\text { Población con necesidades básicas } \\
\text { insatisfechas }\end{array}$ & $\begin{array}{l}\text { Porcentaje de población que presenta al } \\
\text { menos una necesidade básica insatisfecha. }\end{array}$ & Anuario DEIS 2007, 2019 en base a datos censales * \\
\hline
\end{tabular}

APVP: años potenciales de vida perdidos; CIE-10: 10ạ revisión de la Clasificación Internacional de Enfemedades; DEIS: Dirección de Estadísticas e Información en Salud; ENFR: Encuesta Nacional de Factores de Riesgo; IMC: índice de masa corporal.

* Ministerio de Salud de la Nación; Organización Panamericana de la Salud. Indicadores Básicos Argentina. https://www.argentina.gob.ar/salud/deis/ indicadores (accedido el 08/Jul/2020). 
una cola (unilateral), con la finalidad de analizar si el valor medio de los indicadores considerados es significativamente mayor o menor en cada conglomerado respecto a cada uno de los restantes.

Para los análisis descritos, se emplearon los softwares R 3.5.1 (http://www.r-project.org) -y su paquete FactorMineR-y Stata 14 (https://www.stata.com).

En el presente estudio se han considerado los principios éticos para la investigación médica en seres humanos enunciados en la Declaración de Helsinki. Dado que los datos utilizados son de dominio público y no contienen información que permita identificar individuos, la aprobación por un Comité de Ética no fue requerida.

\section{Resultados}

Entre 2005 y 2018 se registró en Argentina un descenso promedio significativo de la mortalidad infantil $(-29,5 \%)$, los años potenciales de vida perdidos (APVP) por enfermedades cardiovasculares $(-10,5 \%)$, por tumores $(-4,6 \%)$, por causas infecciosas $(-8,6 \%)$ y externas $(-6,2 \%)$, porcentaje de población con bajo nivel de actividad física $(-14,8 \%)$, sin cobertura de salud $(-23,4 \%)$ y con necesidades básicas insatisfechas (NBI) $(-27,6 \%)$, así como un aumento de la prevalencia de diabetes, obesidad y esperanza de vida al nacer $(62,5 \%, 67,7 \%$ y $5,2 \%$, respectivamente) (Tabla 1$)$.

Tabla 1

Medidas de resumen de indicadores según ejes de estudio en el año 2005 y 2018 (escala provincial, $n=24$ ), y diferencias observadas en dicho periodo. Argentina.

\begin{tabular}{|c|c|c|c|c|c|c|c|}
\hline \multirow[t]{2}{*}{ Indicadores } & \multicolumn{2}{|c|}{2005 * } & \multicolumn{2}{|c|}{2018 * } & \multirow[t]{2}{*}{ Valor de $p * *$} & \multicolumn{2}{|c|}{$2005-2018$ * } \\
\hline & Media & DE & Media & DE & & $\begin{array}{c}\text { Diferencia } \\
\text { absoluta (media) }\end{array}$ & $\begin{array}{l}\text { Diferencia } \\
\% \text { (media) }\end{array}$ \\
\hline \multicolumn{8}{|l|}{ Transición epidemiológica } \\
\hline Mortalidad infantil & 13,5 & 3,7 & 9,3 & 2,2 & $<0,01$ & $-4,2$ & $-29,5$ \\
\hline APVP por causas cardiovasculares & 71,5 & 14,1 & 63,7 & 13,5 & $<0,02$ & $-7,9$ & $-10,5$ \\
\hline APVP por tumores & 101,3 & 17,6 & 95,1 & 12,4 & 0,02 & $-6,1$ & $-4,6$ \\
\hline APVP por causas externas & 148,0 & 28,2 & 136,4 & 29,3 & 0,04 & $-11,6$ & $-6,2$ \\
\hline APVP por causas infecciosas & 57,1 & 26,4 & 49,7 & 22,5 & $<0,01$ & $-7,5$ & $-8,6$ \\
\hline Tasa de sífilis congénita & 93,5 & 80,3 & 147,3 & 155,9 & 0,11 & 53,8 & 124,2 \\
\hline $\begin{array}{l}\text { Prevalencia de hipertensión } \\
\text { arterial }\end{array}$ & 35,1 & 3,3 & 35,4 & 5,3 & 0,73 & 0,3 & 1,1 \\
\hline Prevalencia diabetes tipo 2 & 8,3 & 1,6 & 13,0 & 2,1 & $<0,01$ & 4,7 & 62,5 \\
\hline \multicolumn{8}{|l|}{ Transición nutricional } \\
\hline Bajo nivel de actividad física & 55,6 & 10,1 & 46,2 & 11,4 & $<0,01$ & $-9,5$ & $-14,8$ \\
\hline Obesidad en adultos/as & 16,1 & 2,4 & 26,7 & 3,8 & $<0,01$ & 10,7 & 67,7 \\
\hline Bajo peso al nacer & 7,1 & 0,8 & 7,0 & 0,6 & 0,29 & $-0,1$ & $-0,4$ \\
\hline \multicolumn{8}{|l|}{ Indicadores sociodemográficos } \\
\hline Población sin cobertura de salud & 48,9 & 10,9 & 37,9 & 11,0 & $<0,01$ & $-10,9$ & $-23,4$ \\
\hline $\begin{array}{l}\text { Población con necesidades } \\
\text { básicas insatisfecjas }\end{array}$ & 20,1 & 7,8 & 14,4 & 5,5 & $<0,01$ & $-5,7$ & $-27,6$ \\
\hline Esperanza de vida al nacer & 73,3 & 1,5 & 77,1 & 0,9 & $<0,01$ & 3,8 & 5,2 \\
\hline
\end{tabular}

APVP: años potenciales de vida perdidos; DE: desvío estándar;

* Los años se referencian como 2005 y 2018, por ser los extremos de la serie en estudio, pero para algunos indicadores corresponden a 2007 y 2018 , según disponibilidad;

** Test t o prueba de los rangos con signo de Wilcoxon, según corresponda, para las diferencias entre 2005 y 2018 en datos apareados (en negrita: valores de $p$ estadísticamente significativos para $\alpha=0,05$ ). 
Mediante APC, se identificaron tres componentes que acumularon 61,9\% de la varianza total. En la Tabla 2 se distinguen las variables que más contribuyeron a cada componente (aquellas con valor absoluto de coeficiente de correlación $\geq 0,60$ ). Desde el marco de la transición epidemiológica, se interpreta que la primera componente se relaciona positivamente con variables características de estadios más avanzados de transición epidemiológica-nutricional; la segunda componente, con variables relevantes en estadios de doble carga de enfermedad, mientras que la tercera componente, representaría a estadios de transición epidemiológica-nutricional más incipientes. De las variables ilustrativas, sólo la cobertura de salud y esperanza de vida al nacer evidenciaron relación directa con la componente principal 1 (Tabla 2). Seguidamente, en base a la medición de las distancias entre variables en este espacio "epidemiológico-nutricional" construido por el ACP, se identificaron tres agrupamientos (conglomerados de unidades geográficas), que se distinguen según las variables que los caracterizan (aquellas cuya media a nivel del conglomerado resulta diferente a la media nacional, con nivel de significación $\alpha=0,05$ ) (Tabla 3).

En el conglomerado 1 se posicionaron el 25\% $(n=6)$ de las jurisdicciones, abarcando principalmente provincias del área central y patagónica de Argentina, más Ciudad Autónoma de Buenos Aires. El conglomerado 2, concentró 12 provincias (50\%) dispersas en distintas regiones del país, seguido por el conglomerado 3 , conformado por el $25 \%$ restante ( $n=6$ provincias), principalmente localizadas en la región noroeste, más Misiones al este (Tabla 3). La distribución geográfica según conglomerados -que se corresponden con los perfiles de transición-epidemiológica identificados- se ilustra en el mapa de la Figura 1.

Tabla 2

Análisis de componentes principales: coeficientes de correlación de las variables seleccionadas con los componentes principales construidos. Argentina 2005-2018.

\begin{tabular}{|c|c|c|c|}
\hline Variables & Componente 1 & Componente 2 & Componente 3 \\
\hline \multicolumn{4}{|l|}{ Transición epidemiológica } \\
\hline Mortalidad infantil & $-0,70$ & 0,35 & 0,38 \\
\hline APVP por causas cardiovasculares & 0,66 & 0,36 & 0,22 \\
\hline APVP por tumores & 0,81 & $-0,05$ & 0,16 \\
\hline APVP por causas externas & 0,66 & $-0,13$ & 0,37 \\
\hline APVP por causas infecciosas & $-0,22$ & 0,65 & $-0,04$ \\
\hline Tasa de sífilis congénita & $-0,38$ & 0,60 & 0,02 \\
\hline Prevalencia de hipertensión arterial & 0,34 & $-0,38$ & 0,21 \\
\hline Prevalencia diabetes tipo 2 & 0,26 & 0,60 & 0,01 \\
\hline \multicolumn{4}{|l|}{ Transición nutricional } \\
\hline Bajo nivel de actividad física & 0,42 & 0,63 & 0,47 \\
\hline Obesidad en adultos/as & 0,46 & 0,41 & $-0,60$ \\
\hline Bajo peso al nacer & $-0,31$ & $-0,13$ & 0,83 \\
\hline \multicolumn{4}{|l|}{ Indicadores sociodemográficos } \\
\hline Población sin cobertura de salud & 0,66 & $-0,19$ & 0,14 \\
\hline Esperanza de vida al nacer & 0,49 & $-0,05$ & 0,33 \\
\hline Población con necesidades básicas insatisfecjas & $-0,44$ & $-0,19$ & 0,28 \\
\hline Varianza explicada (\%) & 26,55 & 19,87 & 15,43 \\
\hline Varianza explicada acumulada (\%) & 26,55 & 46,43 & 61,86 \\
\hline
\end{tabular}

APVP: años potenciales de vida perdidos; HTA: hipertensión arterial.

Nota: Ias variables corresponden a la diferencia porcentual de los indicadores entre 2005 y 2018; para algunos indicadores corresponden a 2007 y 2018, según disponibilidad. En negrita, variables dominantes en cada componente (valor absoluto del coeficiente de correlación $\geq 0,60$ ). 
Tabla 3

Perfiles de transición epidemiológica-nutricional identificados en Argentina: variables epidemiológico-nutricionales y sociodemográficas que los caracterizan.

\begin{tabular}{|c|c|c|c|}
\hline \multirow[t]{2}{*}{ Perfiles/Variables } & \multicolumn{2}{|c|}{$\%$ de cambio $2005-2018$ * } & \multirow[t]{2}{*}{ Valor de $p * *$} \\
\hline & $\begin{array}{l}\text { Media grupal } \\
\text { (del clúster) }\end{array}$ & $\begin{array}{c}\text { Media global } \\
\text { (nacional) }\end{array}$ & \\
\hline \multicolumn{4}{|c|}{ Reemergencia de enfermedades infecciosas *** } \\
\hline Tasa de sífilis congénita & 401,37 & 124,23 & $<0,001$ \\
\hline Mortalidad infantil & $-15,52$ & $-29,5$ & 0,002 \\
\hline APVP por causas infecciosas & 13,17 & $-8,59$ & 0,018 \\
\hline APVP por causas externas & $-24,9$ & $-6,21$ & 0,018 \\
\hline APVP por tumores & $-16,79$ & $-4,59$ & 0,008 \\
\hline Población sin cobertura de salud & $-31,89$ & $-23,44$ & 0,003 \\
\hline \multicolumn{4}{|c|}{$\begin{array}{l}\text { Persistencia de enfermedades cardiovasculares con mejoras sociosanitarias y en } \\
\text { estilos de vida \# }\end{array}$} \\
\hline Prevalencia de hipertensión arterial & 7,69 & 1,08 & 0,019 \\
\hline APVP por causas infecciosas & $-19,9$ & $-8,58$ & 0,033 \\
\hline Mortalidad infantil & $-34,9$ & $-29,5$ & 0,021 \\
\hline Bajo nivel de actividad física & $-27,23$ & $-14,83$ & 0,010 \\
\hline \multicolumn{4}{|c|}{$\begin{array}{l}\text { Consolidación de la tríada obesidad-sedentarismo-enfermedades } \\
\text { cardiometabólicas \#\# }\end{array}$} \\
\hline Prevalencia de sedentarismo & 9,86 & $-14,83$ & 0,003 \\
\hline APVP por causas cardiovasculares & 1,34 & $-10,52$ & 0,003 \\
\hline Diabetes tipo 2 & 95,16 & 62,53 & 0,012 \\
\hline Obesidad & 86,58 & 67,69 & 0,013 \\
\hline Población sin cobertura de salud & $-17,58$ & $-23,44$ & 0,043 \\
\hline
\end{tabular}

APVP: años potenciales de vida perdidos.

* Los años se referencian como 2005 y 2018, por ser los extremos de la serie en estudio, pero para algunos indicadores corresponden a 2007 y 2018, según disponibilidad;

** Prueba t de Student para la diferencia de medias grupal vs. global (valores p estadísticamente significativos para a = 0,05);

*** Ciudad Autónoma de Buenos Aires y provincias de Córdoba, La Pampa, Río Negro, Santa Cruz y Tierra del Fuego;

\# Provincias de Buenos Aires, Catamarca, Corrientes, Chaco, Chubut, Entre Ríos, Formosa, Mendoza, Neuquén, Santa Fe, San Luis y San Juan;

\#\# Provincias de Jujuy, La Rioja, Misiones, Salta, Santiago del Estero y Tucumán.

El primer perfil (conglomerado 1) fue denominado "reemergencia de las enfermedades infecciosas" dado que entre sus características se destaca el aumento significativo de la sífilis congénita (superior al 400\%, vs. 124\% a nivel nacional) y APVP por enfermedades infecciosas (aumento del 13\%, vs. un descenso nacional en torno al 8\%), acompañadas de un descenso más acentuado que el observado a nivel país en los APVP por tumores y causas externas, y del porcentaje de población sin cobertura de salud. A esto se suma, un descenso de la mortalidad infantil menos acentuado que a nivel nacional (Tabla 3). El segundo perfil (conglomerado 2), por su parte, lo denominamos "persistencia de enfermedades cardiovasculares con mejoras sociosanitarias y en estilos de vida", dado el aumento observado en la prevalencia de hipertensión arterial y un descenso en el porcentaje de población con bajo nivel de actividad física (-27,2\%) mayor que el nacional (-14,8\%, p = 0,010); esto, en el marco de un descenso en los APVP por causas infecciosas y mortalidad infantil (Tabla 3).

Finalmente, el último perfil (conglomerado 3) fue denominado "consolidación de la tríada obesidad-sedentarismo-enfermedades cardiometabólicas”, en tanto se caracteriza por aumento de los APVP por causas cardiovasculares y del porcentaje de población con bajo nivel de actividad física (igual a 1,3\% y 9,9\%, respectivamente), que se contrapone con los descensos medios observados a nivel nacional, y a lo cual acompaña un aumento en la prevalencia de diabetes tipo 2 (del 95\%) y de obesi- 


\section{Figura 1}

Distribución geográfica de los perfiles de transición epidemiológica-nutricional identificados en Argentina, 2005-2018.

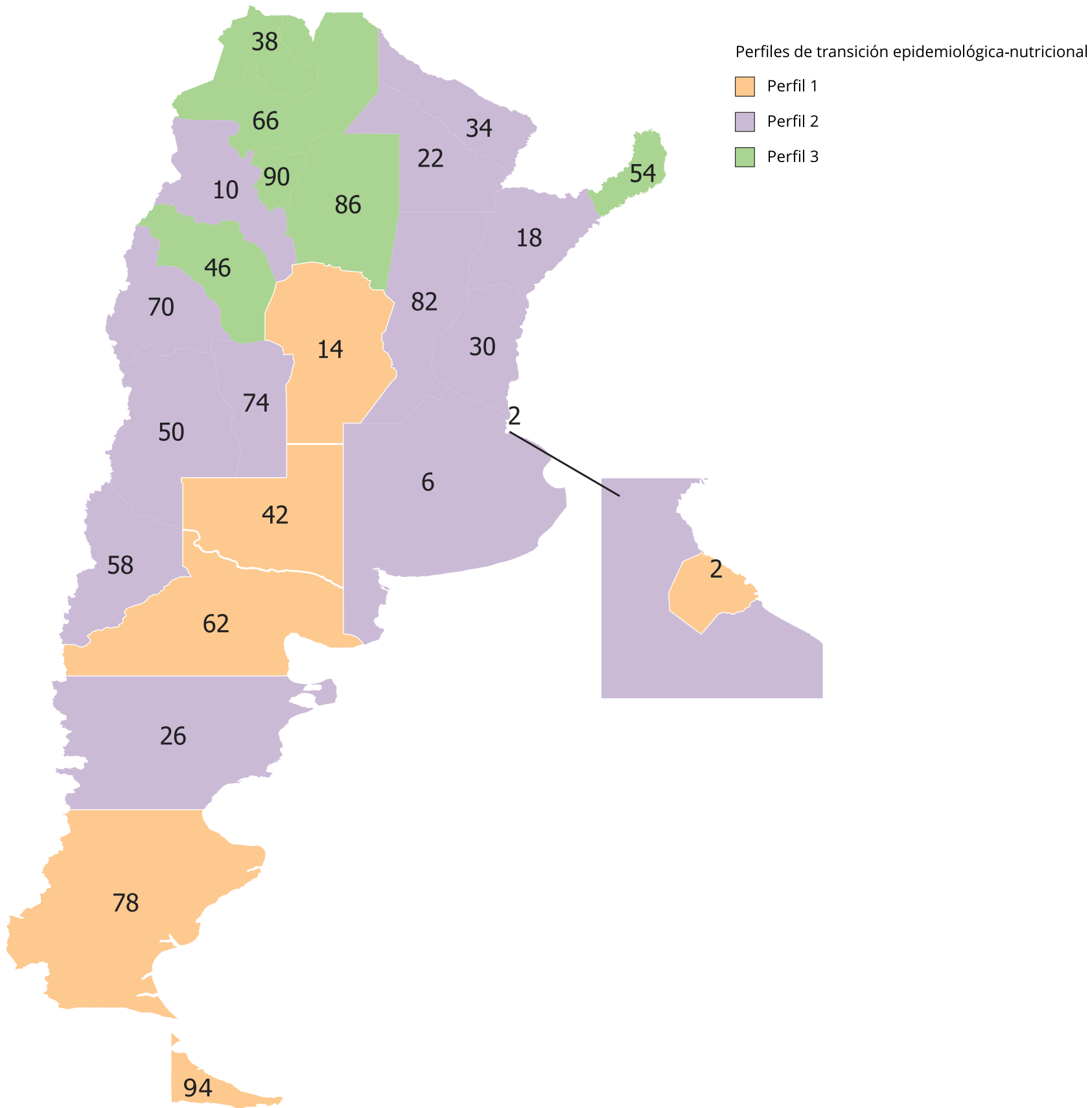

Provincias o unidades geográficas: 2 - Ciudad Autónoma de Buenos Aires; 6 - Buenos Aires; 10 - Catamarca; 14 - Córdoba; 18 - Corrientes; 22 - Chaco; 26 - Chubut; 30 - Entre Ríos; 34 - Formosa; 38 - Jujuy; 42 - La Pampa; 46 - La Rioja; 50 - Mendoza; 54 - Misiones; 58 - Neuquén; 62 - Río Negro; 66 - Salta; 70 - San Juan; 74 - San Luis; 78 - Santa Cruz; 82 - Santa Fe; 86 - Santiago del Estero; 90 - Tucumán; 94 - Tierra del Fuego e Islas del Atántico Sur. Nota: Perfil 1: reemergencia de las enfermedades infecciosas; Perfil 2: persistencia de enfermedades cardiovasculares con mejoras sociosanitarias y en estilos de vida; Perfil 3: consolidación de la tríada obesidad-sedentarismo-enfermedades cardiometabólicas. 
dad (86\%), superiores a los registrados a nivel nacional (Tabla 3). Se observa también un porcentaje de cambio decreciente menos acentuado que en el país en la proporción de población sin cobertura de salud.

Respecto a la morbi-mortalidad por COVID-19 según perfil de transición epidemiológica-nutricional (Tabla 4), se observa que la situación epidemiológica en Argentina es heterogénea entre grupos (perfiles identificados), como intra-grupos (dado las diferencias observadas entre valores máximo y mínimo, y rangos intercuartílicos estimados). En general, se distingue una situación relacionada a COVID-19 más desfavorable en las provincias con perfil "reemergencia de enfermedades infecciosas", excepto para el indicador tasa de letalidad, que concentra valores más altos en el último perfil "consolidación de la tríada obesidad-sedentarismo-enfermedades cardiometabólicas". En el primero, se estimó que las provincias que conforman el conglomerado presentaron en promedio $( \pm \mathrm{DE})$ un total de 4.835,9 $( \pm 2.702)$ casos y 97,1 $( \pm 52,9)$ defunciones por COVID-19, por 100.000 personas, mientras que en los dos perfiles restantes la media para estos indicadores estuvo por debajo de esos valores (entre 2417-1970 casos y 48,5-59,6 defunciones, cada 100.000 habitantes). Al analizar la diferencia entre medias para cada indicador (Tabla 4), se observó que existieron diferencias significativas (a un nivel $\alpha=0,05$ ) entre conglomerados (perfiles). La incidencia acumulada promedio fue mayor en las provincias con perfil "reemergencia de enfermedades infecciosas" (media $=4835,9$ casos $/ 100.000$ ), en comparación al promedio estimado en aquellas que conforman el perfil "persistencia de enfermedades cardiovasculares con mejoras sociosanitarias y en estilos de vida" ( media $=2417,0 ; p=0,0159$ ), y el de "consolidación de la tríada obesidad-sedentarismo-enfermedades cardiometabólicas" (media = 1970,3; $\mathrm{p}=0$,0205). Además, la mortalidad acumulada por COVID-19 también fue superior en el primer grupo (media $=97,1$ defunciones cada 100.000 personas), en relación al segundo (media $=48,5 ; \mathrm{p}$ $=0,0187$ ). La tasa de letalidad, en cambio, resultó mayor entre las provincias del perfil "consolidación de la tríada obesidad-sedentarismo-enfermedades cardiometabólicas" versus las que conformaron el perfil "persistencia de enfermedades cardiovasculares con mejoras sociosanitarias y en estilos de vida" (media $=2,9 \%$ y $1,9 \%$, respectivamente; $\mathrm{p}=0,0307$ ).

\section{Discusión}

En este estudio se identificaron tres agrupamientos (conglomerados geográficos), correspondientes a distintos perfiles de transición epidemiológica-nutricional en Argentina. La reciente situación de morbi-mortalidad por COVID-19 tiende a ser distinta según estos perfiles. El perfil "reemergencia de enfermedades infecciosas" presentó la situación más desfavorable en términos de incidencia y mortalidad acumuladas, en tanto que el perfil "consolidación de la tríada obesidad-sedentarismoenfermedades cardiometabólicas” presentó la mayor tasa de letalidad.

El perfil "reemergencia de enfermedades infecciosas" interpretamos que estaría en una etapa conocida como "de contra transición" 6,17, con descenso de enfermedades no transmisibles y reemergencia de las infecciosas. Además, su concomitancia con una reducción de la proporción de personas sin cobertura de salud sugiere que estas transformaciones epidemiológico-nutricionales estarían acompañadas de una mejora en las condiciones sociales, mediadas por la situación ocupacional. De hecho, en Argentina la cobertura de salud se organiza, principalmente, en torno a las obras sociales nacionales y provinciales 18 generalmente ligadas a la condición de ocupación laboral.

El perfil "persistencia de enfermedades cardiovasculares con mejoras sociosanitarias y en estilos de vida" se condice con el tercer estadio de la transición epidemiológica propuesta por Omran ${ }^{9}$ (p. 102): "etapa de las enfermedades degenerativas y producidas por el hombre". Asimismo, dada la reducción observada en el porcentaje de población con bajo nivel de actividad física, existen similitudes con un estadio avanzado de la transición nutricional, denominado "cambios conductuales" 3 (p. 139), caracterizado por cambios hacia estilos de vida más saludables 3 .

El último perfil fue llamado "consolidación de la tríada obesidad-sedentarismo-enfermedades cardiometabólicas" por presentar un aumento de los APVP por causas cardiovasculares, porcentaje de población con bajo nivel de actividad física, obesidad y diabetes. Este agrupamiento también estaría en consonancia con el tercer estadio de transición epidemiológica antes mencionado, aunque atravesando una etapa menos avanzada de transición nutricional, siendo que aún no se ha iniciado 
Tabla 4

Indicadores de carga de morbi-mortalidad por COVID-19: medidas resumen según perfiles de transición epidemiológica-nutricional. Argentina, 2020.

\begin{tabular}{|c|c|c|c|c|c|c|}
\hline \multirow[t]{2}{*}{ Medidas resumen } & \multirow{2}{*}{$\begin{array}{c}\text { Perfil } 1 \\
\text { Reemergencia de } \\
\text { enfermedades } \\
\text { infecciosas * }\end{array}$} & \multirow{2}{*}{$\begin{array}{c}\text { Perfil } 2 \\
\text { Persistencia de } \\
\text { enfermedades } \\
\text { cardiovasculares } \\
\text { con mejoras } \\
\text { sociosani-tarias } \\
\text { y en estilos de } \\
\text { vida ** }\end{array}$} & \multirow{2}{*}{$\begin{array}{c}\text { Perfil } 3 \\
\text { Consolidación } \\
\text { de la triada } \\
\text { obesidad- } \\
\text { sedentarismo- } \\
\text { enfermedades } \\
\text { cardiome- } \\
\text { tabólicas *** }\end{array}$} & \multicolumn{3}{|c|}{$\begin{array}{c}\text { Comparación de medias \# } \\
\text { (valor de } p \text { ) }\end{array}$} \\
\hline & & & & Perfil 1 vs. 2 & Perfil 1 vs. 3 & Perfil 2 vs. 3 \\
\hline Tasa de incidencia acumulada \#\# por & & & & $1>2$ & $1>3$ & $2>3$ \\
\hline COVID-19 (por 100.000 personas) & & & & & & \\
\hline Media & $4.835,9$ & $2.417,0$ & $1.970,3$ & 0,0159 & 0,0205 & 0,2887 \\
\hline $\mathrm{DE}$ & $2.702,0$ & $1.683,3$ & $1.287,5$ & & & \\
\hline Valor mínimo & 1.768 & 31 & 46 & & & \\
\hline Valor máximo & 9.718 & 5.287 & 3.974 & & & \\
\hline $\begin{array}{l}\text { Mortalidad acumulada \#\# por COVID-19 } \\
\text { (por } 100.000 \text { personas) }\end{array}$ & & & & $1>2$ & $1>3$ & $2<3$ \\
\hline Media & 97,1 & 48,5 & 59,6 & 0,0187 & 0,0999 & 0,2858 \\
\hline $\mathrm{DE}$ & 52,9 & 37,3 & 40,9 & & & \\
\hline Valor mínimo & 26 & 0,5 & 0,7 & & & \\
\hline Valor máximo & 171 & 121 & 110 & & & \\
\hline Tasa de letalidad por COVID-19 (\%) & & & & $1>2$ & $1<3$ & $2<3$ \\
\hline Media & 2,0 & 1,9 & 2,9 & 0,3376 & 0,1145 & 0,0307 \\
\hline $\mathrm{DE}$ & 0,7 & 0,7 & 1,6 & & & \\
\hline Valor mínimo & 1,4 & 0,8 & 1,2 & & & \\
\hline Valor máximo & 3,3 & 3,4 & 4,7 & & & \\
\hline
\end{tabular}

DE: desvío estándar.

Nota: en negrita, diferencias de medias estadísticamente significativas, a un nivel a =0,05.

* Ciudad Autónoma de Buenos Aires y provincias de Córdoba, La Pampa, Río Negro, Santa Cruz y Tierra del Fuego;

** Provincias de Buenos Aires, Catamarca, Corrientes, Chaco, Chubut, Entre Ríos, Formosa, Mendoza, Neuquén, Santa Fe, San Luis y San Juan;

*** Provincias de Jujuy, La Rioja, Misiones, Salta, Santiago del Estero y Tucumán.

\# Prueba t de Student;

\#\# Medida acumulada a la semana epidemiológica 50, al 8 de Diciembre de 2020.

el cambio conductual que se describió anteriormente. Por el contrario, reconocemos similitudes con el estadio denominado "enfermedades no transmisibles relacionadas a la nutrición" 3 (p. 139), que conlleva hábitos sedentarios y modos de comer que determinan una mayor ocurrencia de obesidad y enfermedades crónicas 3 .

La estrecha correspondencia de las tendencias epidemiológicas y nutricionales aquí observada revelan la existencia de no una sino varias transiciones epidemiológicas-nutricionales en Argentina. Esto podría entenderse dentro del marco propuesto por Frenk 6, quien realiza ajustes al planteamiento de Omran 9 , postulando que los estadios pueden superponerse en un mismo país y que la secuencia cronológica no siempre es lineal. En este sentido, y particularmente para la región latinoamericana, se advirtió la existencia de una doble carga de enfermedades transmisibles y no transmisibles, y el desarrollo de una polarización epidemiológica peculiar, evidente no solo entre los países de la región sino, dentro de ellos, entre distintas zonas geográficas y clases sociales 19,20. Similarmente, también en países en desarrollo se ha reportado una gran heterogeneidad en los tiempos, patrones y tendencias del proceso de transición nutricional y epidemiológico 12,21,22,23.

A nivel nacional, Carbonetti et al. ${ }^{24}$ proponen que las condiciones económicas y sociales en Argentina, así como las políticas de salud generaron un contexto epidemiológico para que, en el 
momento de inicio de la transición epidemiológica, sus regiones tuvieran condiciones de salud distintas. Además, otros estudios referidos a la transición nutricional 13, segunda transición demográfica y transición sanitaria 25 reportaron también simultaneidad de procesos transicionales en Argentina. Si bien esos trabajos carecen de una perspectiva temporal como el presente, queda revelada -desde diferentes perspectivas- la heterogeneidad de las dinámicas transicionales en el país, lo cual es congruente con las considerables disparidades socioeconómicas que caracterizan también al territorio nacional, y al ya reportado rol de los determinantes sociales macro-contextuales en la configuración de las transiciones poblacionales 26,27 .

En este estudio se describió, además, la carga de COVID-19 en Argentina. Como tendencia, se distingue una situación comparativamente más desfavorable en incidencia por COVID-19 para el grupo de provincias con perfil de "reemergencia de enfermedades infecciosas", y en cuanto a letalidad para el grupo con perfil "consolidación de la tríada obesidad-sedentarismo-enfermedades cardiometabólicas”. Esto es congruente con la literatura científica que establece una mayor severidad de COVID-19 en las personas con diagnóstico de obesidad y/o diabetes 15,16,28. La obesidad es un estado inflamatorio con una activación crónica del sistema inmunitario, y con afectaciones de las funciones inmunitarias que determinan altas tasas de infecciones y complicaciones 16 . También se ha indicado que la inflamación asociada a la diabetes podría incrementar tanto la morbilidad como la mortalidad por COVID-19 28.

Es interesante notar que el perfil correspondiente a la tríada obesidad-sedentarismo-enfermedades cardiometabólicas aglomera a provincias del noroeste argentino y Misiones, las cuales presentan una situación socioeconómica relativa desfavorable en el marco nacional 29. Esto se confirma, en parte, con la tendencia observada en este grupo respecto al indicador cobertura de salud. Estos hallazgos sugieren que la confluencia de una situación epidemiológica-nutricional de riesgo para COVID-19 (alta prevalencia de obesidad y diabetes) y de condiciones socioeconómicas (y con ellas, posiblemente sociosanitarias) desfavorables podría resultar en peores resultados de salud en esta epidemia. Es posible que en estas poblaciones el peor pronóstico y resultado de los casos de COVID-19, ligados a las patologías prevalentes de base, se vea potenciado por una respuesta ineficiente del sistema de salud ante esta epidemia.

La mayor incidencia de COVID-19 en las provincias con "reemergencia de enfermedades infecciosas" hipotetizamos que podría vincularse a sus condiciones sociodemográficas basales, como la alta urbanización de algunas jurisdicciones que integran este conglomerado, particularmente Ciudad Autónoma de Buenos Aires y Córdoba (se estima que en 2020 sólo estas dos aglutinan el 15\% de la población total de Argentina). En efecto, fue señalado que el fenómeno de urbanización creciente aumenta la exposición a enfermedades infecciosas e impone desafíos para su control especialmente ante situaciones de crisis, como la desatada por COVID-19 30. Se destaca además que este grupo aglomera a la mayoría de las jurisdicciones con la mejor situación socioeconómica del país 29 . El hecho de que, aun registrando el mayor número de casos y muertes por COVID-19 del país, reporte la mejor situación de letalidad, aporta un argumento más a favor del central rol que parece jugar el contexto socioeconómico, y en relación a este, la respuesta del sistema de salud en la gestión de esta epidemia.

Particularmente en la región latinoamericana, se ha alertado que esta pandemia se superpone a desafíos en salud preexistentes, incluyendo alta carga de enfermedades no transmisibles 31 . La importancia de superar la dicotomía enfermedades agudas vs. crónicas en el diseño de programas de salud, ya había sido destacada previamente 32, y se torna aún más necesaria en tiempos de pandemia y en nuestro contexto, donde tradicionalmente los programas que abordan estas enfermedades trabajan aisladamente.

Este trabajo presenta ciertas limitaciones. Primero, al tratarse de un estudio ecológico (unidades de observación geográficas) se recomienda interpretar los resultados con cautela; lo que se observa a nivel agregado no necesariamente representa lo acontecido a nivel individual. Además, se reconoce como debilidad la utilización de fuentes de datos secundarias con cierto desfasaje temporal, aunque consideramos que este no es grande y es la mejor información disponible en nuestro contexto para el periodo de estudio. Por último, la imposibilidad de controlar la heterogeneidad existente en el interior del territorio nacional, en cuanto a calidad y completitud de datos sobre COVID-19, en especial en relación al número de testeos y demoras en el sistema de registro, es otra limitación que pudiera afectar la interpretación relativa a la morbi-mortalidad por COVID-19. 
Concluyendo, la coexistencia de diversos perfiles epidemiológico-nutricionales en Argentina, que parecen presentar situaciones diferenciales en cuanto al impacto de la pandemia por COVID-19, sugiere la necesidad de adoptar abordajes integrales en la definición de políticas tendientes a mejorar la salud de la población, reconociendo lo imbricados que están los procesos epidemiológicosnutricionales y crónicos-agudos. Esa integración, no obstante, debiera considerar que esas articulaciones acontecen en un escenario heterogéneo, tanto socioeconómica como sanitariamente, lo que determina necesidades de acción contexto-específicas. En consonancia con lo propuesto por la Comisión Económica para América Latina y el Caribe (CEPAL), un esfuerzo adicional en materia política y social será requerido para reducir las inequidades sociales en salud preexistentes, que se verán acentuadas tras los graves efectos económicos y sociales causados por la pandemia, en especial en los países latinoamericanos 33 .

\section{Colaboradores}

G. F. Scruzzi y S. A. Pou han contribuido en la concepción y diseño del estudio, análisis e interpretación de los datos, redacción del artículo, revisión del contenido intelectual del mismo, y aprobación de la versión final del manuscrito. N. Tumas contribuyó en la revisión crítica del manuscrito con importantes aportes de contenido intelectual, en la interpretación de datos y redacción del artículo, y aprobación de la versión final del artículo para su publicación.

\section{Informaciones adicionales}

ORCID: Graciela Fabiana Scruzzi (0000-00023187-5112); Natalia Tumas (0000-0003-47306624); Sonia Alejandra Pou (0000-0002-85719318).

\section{Agradecimientos}

Agradecemos a los integrantes del proyecto Epidemiología de las Enfermedades no Transmisibles y Obesidad en Argentina: Análisis de su Relación con el Proceso de Transición Nutricional desde una Perspectiva EspacioTemporal, de la Universidad Nacional de Córdoba, por su colaboración en la obtención y procesamiento de datos, a partir de fuentes secundarias utilizadas en el presente trabajo. A la Secretaria de Ciencia y Técnica, Universidad Nacional de Córdoba (Cod. 30820150100344CB; 3620190100084CB01).

\section{Referencias}

1. Thompson WS. Population. AJS 1929; 34:95975.

2. Omran AR. The epidemiologic transition: a theory of the epidemiology of population change. 1971. Milbank Q 2005; 83:731-57.

3. Popkin BM. Nutritional patterns and transitions. Popul Dev Rev 1993; 19:138-57.

4. Olshansky SJ, Ault AB. The fourth stage of the epidemiologic transition: the age of delayed degenerative diseases. Milbank Q 1986; 64:355-91.

5. Frenk J, Bobadilla JL, Sepulveda J, De Cervantes ML. Health transition in middle-income countries: new challenges for health care. Health Policy Plan 1989; 4:29-39.

6. Frenk J, Freika T, Bobadilla J, Stern C, Lozano $\mathrm{R}$, Sepúlveda J, et al. La transición epidemiológica en América Latina. Bol Oficina Sanit Panam 1991; 111:485-96.

7. Caldwell JC. Health transition: the cultural, social and behavioural determinants of health in the Third World. Soc Sci Med 1993; 36:12535.

8. Mackenbach JP. The epidemiologic transition theory. J Epidemiol Community Health 1994; 48:329-31

9. Omran AR. The epidemiologic transition theory revisited thirty years later. World Health Stat Q 1998; 53:99-119.

10. Popkin BM. The nutrition transition in lowincome countries: an emerging crisis. Nutr Rev 1994; 52:285-98. 
11. Popkin BM. Contemporary nutritional transition: determinants of diet and its impact on body composition. Proc Nutr Soc 2011; 70:8291.

12. Popkin BM, Gordon-Larsen P. Nutrition transition: worldwide obesity dynamics and their determinants. Int J Obes Relat Metab Disord 2004; 28 Suppl 3:S2-9.

13. Tumas N, Rodríguez Junyent C, Aballay LR, Scruzzi GF, Pou SA. Nutrition transition profiles and obesity burden in Argentina. Public Health Nutr 2019; 22:2237-47.

14. Durán P. Transición epidemiológica nutricional o el "efecto mariposa". Arch Argent Pediatr 2005; 103:195-7.

15. Popkin BM, Du S, Green WD, Beck MA, Algaith $\mathrm{T}$, Herbst $\mathrm{CH}$, et al. Individuals with obesity and COVID-19: a global perspective on the epidemiology and biological relationships. Obes Rev 2020; 21:e13128.

16. Vas P, Hopkins D, Feher M, Rubino F, Whyte MB. Diabetes, obesity and COVID-19: a complex interplay. Diabetes Obes Metab 2020; 22:1892-6

17. Alvis Guzmán NR, De La Hoz Restrepo F. Tendencias de la mortalidad por enfermedades infecciosas en Cartagena de Indias, Colombia, 1995-2000: una medida de la transición epidemiológica. Rev Cienc Salud (Bogotá) 2004; 6:235-52.

18. Organización Panamericana de la Salud. Informe de país Argentina. https:// www.paho.org/salud-en-las-americas2017/?page_t_es=informes\%20de\%20pais/ argentina\&lang=es (accedido el 06/Nov/2020).

19. Delgado J. La urbanización difusa, arquetipo territorial de la ciudad-región. Sociológica 2003; 18:13-48.

20. Centro Latinoamericano y Caribeño de Demografía. Población y salud en América Latina y el Caribe: retos pendientes y nuevos desafíos. Santiago de Chile: Naciones Unidas; 2010.

21. Barría RM, Amigo H. Transición nutricional: una revisión del perfil latinoamericano. Arch Latinoam Nutr 2006; 56:3-11.

22. Mendoza W, Miranda JJ. Global shifts in cardiovascular disease, the epidemiologic transition, and other contributing factors: toward a new practice of global health cardiology. Cardiol Clin 2017; 35:1-12.

23. Ramos AD, Montesinos FF, Letucet EL, Santana CV, Tortoló IS. Transición epidemiológica. Rev Medica Electron 2003; 25:36-42.
24. Carbonetti A. Historia de una epidemia olvidada: la pandemia de gripe española en la argentina, 1918-1919. Desacatos 2010; (32):159-74.

25. Fanta Garrido J, Tumas N. Sincronicidades entre la transición sanitaria y la segunda transición demográfica en Argentina durante la primera década del siglo XXI. Revista Latinoamericana de Población 2020; 14:257-95.

26. McKeown RE. The epidemiologic transition: changing patterns of mortality and population dynamics. Am J Lifestyle Med 2009; 3(1 Suppl):19S-26S.

27. Dyson T. Population and development: the demographic transition. London: Zed Books Ltd; 2013.

28. Hussain A, Bhowmik B, Do Vale Moreira NC. COVID-19 and diabetes: knowledge in progress. Diabetes Res Clin Pract 2020; 162:108142.

29. Velázquez G. Geografía y calidad de vida en Argentina: análisis regional y departamental (2010). Tandil : Universidad Nacional del Centro de la Provincia de Buenos Aires; 2016.

30. Connolly C, Ali SH, Keil R. On the relationships between COVID-19 and extended urbanization. Dialogues Hum Geogr 2020; 10:213-6.

31. Diez Roux AV, Barrientos-Gutierrez T, Caiaffa WT, Miranda JJ, Rodriguez D, Sarmiento OL, et al. Urban health and health equity in Latin American cities: what COVID-19 is teaching us. Cities \& Health 2020; Special Issue: COVID-19. https://www.tandfonline.com/ doi/full/10.1080/23748834.2020.1809788.

32. Oni T, Unwin N. Why the communicable/noncommunicable disease dichotomy is problematic for public health control strategies: implications of multimorbidity for health systems in an era of health transition. Int Health 2015; 7:390-9.

33. Comisión Económica para América Latina y el Caribe. Pactos políticos y sociales para la igualdad y el desarrollo sostenible en América Latina y el Caribe en la recuperación posCOVID-19. Informe Especial COVID-19 2020; (8). https://repositorio.cepal.org/bits tream/handle/11362/46102/4/S2000673_ es.pdf.

34. Ministerio de Salud de la Nación. Primera Encuesta Nacional de Factores de Riesgo. Buenos Aires: Ministerio de Salud de la Nación; 2006.

35. Instituto Nacional de Estadística y Censos. 4a Encuesta Nacional de Factores de Riesgo: resultados definitivos. Buenos Aires: Instituto Nacional de Estadística y Censos; 2019. 
Abstract

The study aimed to identify epidemiologicalnutritional profiles in Argentina and to compare the burden of morbimortality from COVID-19. A multigroup ecological study was conducted with 24 geographic units in Argentina. We estimated the percent change from 2005 to 2018 in indicators of the epidemiological-nutritional transition and sociodemographic indicators according to geographic unit. We performed principal components analysis and hierarchical cluster analysis to identify geographic groupings to define profiles in the epidemiological-nutritional transition. By group, we calculated summary measures of COVID-19 cumulative incidence (CI), mortality, and case fatality (epidemiological week 50), establishing comparisons via Student's t test. Three profiles were identified: (1) reemergence of infectious diseases, (2) persistence of cardiovascular diseases despite social, health, and lifestyle improvements, and (3) consolidation of the triad obesity-sedentarismcardiometabolic diseases. Mean COVID-19 cumulative incidence and mortality were higher in provinces with profile 1 compared to profile 2 (CI: $p=0.0159$; mortality: $p=0.0187$ ) and profile 3 (CI: $p=0.0205)$. Case-fatality was higher in profile 3 , which includes provinces with more unfavorable socioeconomic conditions, showing significant differences from profile $2(p=0.0307)$. In conclusion, there are distinct epidemiologicalnutritional profiles in Argentina which tend to differ in terms of their COVID-19 epidemiological situation. Strategies to fight COVID-19 should consider the underlying epidemiological, nutritional, and sociodemographic characteristics.

Coronavirus Infections; Nutritional Transition; Population Dynamics; Social Epidemiology

\section{Resumo}

O objetivo deste trabalho era identificar perfis epidemiológico-nutricionais na Argentina, $e$ comparar a sua carga de morbimortalidade por COVID-19. Foi desenvolvido um estudo ecológico multigrupal (24 unidades geográficas da Argentina). Foi avaliado o percentual de mudança 20052018 de indicadores de transição epidemiológica-nutricional, e sociodemográficos, por unidade geográfica. Foi feita uma análise de componentes principais e de cluster hierárquico para identificar agrupamentos geográficos, definindo perfis de transição epidemiológica-nutricional. Por grupo, foram calculadas medidas de resumo de incidência acumulada (IA), mortalidade e taxa de letalidade por COVID-19 (semana epidemiológica 50), estabelecendo comparações mediante teste tde Student. Foram identificados três perfis: (1) reemergência de doenças infecciosas, (2) persistência de doenças cardiovasculares com melhorias sociossanitárias e nos estilos de vida, e (3) consolidação da tríade obesidade-sedentarismo-doenças cardiometabólicas. A IA e a mortalidade média por COVID-19 foi maior nas províncias com perfil 1, comparado com o perfil 2 (IA: $p=0,0159$; mortalidade: $p=0,0187)$ e o perfil 3 (IA: $p=0,0205)$. A letalidade resultou maior no perfil 3 , que abrange provincias com situação socioeconômica mais desfavorável, sendo encontradas diferenças significativas com relação ao perfil $2(p=0,0307)$. Em conclusão, existem distintos perfis epidemiológico-nutricionais na Argentina, que tendem a diferenciar-se na sua situação epidemiológica de COVID-19. As estratégias contra a COVID-19 deveriam considerar as características epidemiológico-nutricionais e sociodemográficas de base.

Infecções por Coronavírus; Transição Nutricional; Dinâmica Populacional; Epidemiologia Social
Recibido el 14/Dic/2020

Versión final presentada el 16/Abr/2021

Aprobado el 29/Abr/2021 